\title{
A kinetic model improves off-target predictions and reveals the physical basis of SpCas9 fidelity
}

Behrouz Eslami-Mossallam ${ }^{1, *}$, Misha Klein ${ }^{1, *}$, Constantijn v.d. Smagt ${ }^{1}$, Koen v.d. Sanden ${ }^{1}$, Stephen K. Jones Jr., $^{2,3,4}$ John A. Hawkins, ${ }^{2,3,4,5}$, Ilya J. Finkelstein ${ }^{2,3,4}$, and Martin Depken ${ }^{1, * *}$

${ }^{1}$ Kavli Institute of NanoScience and Department of BioNanoScience, Delft University of Technology, Delft $2629 \mathrm{HZ}$, the Netherlands

${ }^{2}$ Department of Molecular Biosciences, University of Texas at Austin, Austin, Texas 78712, USA

${ }^{3}$ Institute for Cellular and Molecular Biology, University of Texas at Austin, Austin, Texas 78712, USA

${ }^{4}$ Center for Systems and Synthetic Biology, University of Texas at Austin, Austin, Texas 78712, USA

${ }^{5}$ Oden Institute for Computational Engineering and Science, University of Texas at Austin, Austin, Texas 78712, USA

* equal contribution

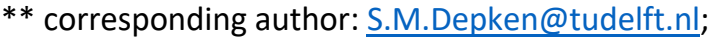

The $S$. pyogenes $(S p)$ Cas9 endonuclease is an important gene-editing tool. SpCas9 is directed to target sites via a single guide RNA (sgRNA). However, SpCas9 also binds and cleaves genomic off-target sites that are partially matched to the sgRNA. Here, we report a microscopic kinetic model that simultaneously captures binding and cleavage dynamics for SpCas9 and Sp-dCas9 in free-energy terms. This model not only outperforms state-of-the-art off-target prediction tools, but also details how Sp-Cas9's structure-function relation manifests itself in binding and cleavage dynamics. Based on the biophysical parameters we extract, our model predicts SpCas9's open, intermediate, and closed complex configurations and indicates that Rloop progression is tightly coupled with structural changes in the targeting complex. We show that SpCas9 targeting kinetics are tuned for extended sequence specificity while maintaining on-target efficiency. Our extensible approach can characterize any CRISPR-Cas nuclease - benchmarking natural and future highfidelity variants against SpCas9; elucidating determinants of CRISPR fidelity; and revealing pathways to increased specificity and efficiency in engineered systems.

CRISPR-Cas9 (Clustered Regularly Interspaced Short Palindromic Repeats - CRISPR associated protein 9) is a ubiquitous tool in the biological sciences ${ }^{1,2}$ with applications ranging from live-cell imaging ${ }^{3}$ and gene knockdown/overexpression ${ }^{4,5}$, to genetic engineering ${ }^{6,7}$ and gene therapy ${ }^{8,9}$. Streptococcus pyogenes (Sp) Cas 9 is programmed with a $\sim 100$ nucleotide (nt) single-guide RNA (sgRNA) to target DNAs based on the level of complementarity to a $20 \mathrm{nt}$ segment of the sgRNA ${ }^{10}$. Wild type SpCas9 (Cas9 from now on) induces specific double-stranded breaks and the catalytically 'dead' Cas9 (dCas9) mutants allow for binding the target DNA without cleavage ${ }^{3,5}$. Apart from complimentary on-targets, Cas9-sgRNA also binds and cleaves partiallycomplementary off-target DNA sites ${ }^{11-18}$. Off-target cleavage risks unwanted genomic alterations, including point mutations, large-scale deletions, and chromosomal rearrangements ${ }^{19}$. The potentially deleterious effects associated with such editing errors impedes wide-spread implementation of the CRISPR toolkit in human therapeutics.

Off-target sites are identified in silico by a growing set of prediction tools. These tools use bioinformatics ${ }^{20,21}$, machine learning ${ }^{22,23}$, and heuristic ${ }^{12,14,24,25}$ approaches to rank genomic sites based on their own unique offtarget activity scores. However, none of these tools attempt to model the microscopic kinetic properties that govern Cas9-DNA binding and nuclease activation. This quantitative kinetic modeling is essential for understanding how in vivo Cas9 activity depends on enzyme concentration and exposure time. Both of these parameters are frequently exploited by experimentalists to limit off-target activity in cells ${ }^{26}$.

Quantitative predictions of Cas9 activity requires a physical model that accounts for the kinetic nature of the problem. Existing physical models ${ }^{24,27}$ implicitly assume that Cas9-sgRNA binding equilibrium is reached over the entire genome before DNA cleavage. However, binding does not necessarily equilibrate before cleavage $e^{28,29}$, as can be inferred from the fact that binding and cleavage correlate weakly in vitro and in cells ${ }^{30-}$ 
${ }^{32}$ (see below). Here, we construct a comprehensive kinetic model that includes binding and cleavage reactions, and globally train it on two high-throughput in vitro datasets that capture each process separately ${ }^{15}$. Our fully parameterized model accurately predicts an independent high-throughput dataset ${ }^{11}$, without the use of any additional fitting parameters. Our model is parameterized in terms of physical quantities and therefore offers insights into biophysical mechanisms. By establishing the free-energy landscape of the targeting reaction with any off-target, which shows that the difference in binding and cleavage activities ${ }^{30-39}$ stems from a (relatively) long-lived DNA-bound intermediate. We further show that this state is tuned for both high cleavage specificity and on-target cleavage efficiency. We also connect the binding intermediate to the intermediate $\mathrm{HNH}$ conformation observed in single-molecule FRET experiments ${ }^{40,41}$, and argue that the conformational change is driven by R-loop formation. Finally, we show that our kinetic model outperforms the two best-performing genomic off-target prediction tools used today ${ }^{12,24,42}$.

\section{Results}

\section{Kinetic model simultaneously captures binding and cleavage profiles}

Figure 1a describes the microscopic kinetic schema that underpins our physical Cas9 binding and cleavage model. First, the Cas9-sgRNA ribonucleoprotein complex recognizes a 3nt protospacer adjacent motif (PAM) DNA sequence-canonically 5'-NGG-3'-via protein-DNA interactions ${ }^{43,44}$. Binding to the PAM sequence opens the DNA double helix, and allows the first base of the target sequence to hybridize with the sgRNA ${ }^{43,44}$. The DNA double helix further denatures as the sgRNA and target strand form an RNA-DNA hybrid (R-loop) ${ }^{45-48}$. The $\mathrm{R}$-loop grows and shrinks in single nucleotide steps until it is either reversed and Cas 9 dissociates, or it reaches completion (a 20 nt hybrid). If the R-loop reaches completion, Cas9 uses its $\mathrm{HNH}$ and RuvC nuclease domains to cleave both strands of the DNA duplex ${ }^{49}$.

The most general reaction schemes for cleavage and binding are completely parameterized only when we estimate all the rates shown in Fig. 1a for every potential guide-target combination-a prohibitively large number of parameters for any genome. To render parameter estimation tractable, we make four mechanistic model assumptions: (1) mismatch positions within the hybrid are more important than mismatch types-e.g. G-G vs. G-A (as can be inferred directly from data ${ }^{11,15}$ ), and all 12 mismatch types can be treated equally; (2) dCas9 differs from Cas9 only in that dsDNA bond-cleavage catalysis is completely suppressed, and all other rates can be taken to be identical between the two 40,$50 ;(3)$ a mismatch influences only the reversal of the mismatched base pairing, leaving all other rates unchanged; (4) all hybrid-bond-formation rates are equal, and independent of complementarity. These assumptions are justified post hoc by showing that the targeting dynamics are completely determined by even a much smaller set of effective rates. Though our model is kinetic, we can use the detailed-balance condition for microscopic rates (Supplementary Information) to define the free-energy of each state in our model (Fig. 1b). Our model assumptions reduce the total number of parameters to 44: the (concentration dependent) rate of PAM binding from solution $\left(k_{\mathrm{on}}\right)$ and the associated free-energy cost; a single internal forward (bond-formation) rate $\left(k_{\mathrm{f}}\right) ; 20$ free-energy costs dictating R-loop progression for matching guide and target; 20 free-energy penalties for mismatches at different R-loop positions; and, for Cas 9 , the rate at which the final cleavage reaction is catalyzed $\left(k_{\text {cat }}\right)$ (see Supplementary Information for further details). When extending the R-loop, both gains and losses in free-energy are possible as base-pairing interactions, protein-DNA interactions ${ }^{50}$, and any induced conformational changes $40,41,49,51$ all contribute to the stability of the Cas9-sgRNA-DNA complex. As we assume that mismatches only facilitate the reversal of the mismatched base pairs, the entire free-energy landscape will rise by a positive amount from the mismatch onwards (c.f. pink and blue free-energy landscapes in Fig. 1b).

We used three high-throughput assays to train and validate our kinetic model. The first training data set estimates the effective cleavage rates $\left(k_{\text {clv }}\right)$ for a library of off-target DNA sequences by monitoring the fraction of uncut DNA over time ${ }^{15}$ (NucleaSeq in Fig. 1c; Supplementary Information). The second training data set reports on the effective association constant $\left(K_{\mathrm{A}}\right)$ over a library of off-target DNA sequences exposed to dCas9sgRNA for 10 min $^{15,52}$ (CHAMP in Fig. 1d; Supplementary Information). The third data set, used for validating the model, reports the effective association rate estimated over 1500 seconds of exposure to dCas9-sgRNA at $1 \mathrm{nM}$ concentration (HiTS-FLIP in Fig. 1e; Supplementary Information) ${ }^{11}$. Our kinetic model describes all these experiments even though each dataset uses either Cas9 or dCas9 to report on the cleavage rates, association constants, or association rates by sweeping either concentration or time ${ }^{28}$.

We trained the kinetic model on DNA binding (CHAMP) and cleavage (NucleaSeq) datasets collected using the same sgRNA and mismatched target DNA library ${ }^{15}$. The parameters were globally fit to the binding affinities 
and cleavage rates for all off-target DNA sequences with up to two mismatches. The rates from different types of mismatches were averaged together (Supplementary Information). Although these two datasets do not correlate well with each other directly (55\%, see Supplementary Figure 1a), our model reproduces effective cleavage rates (Fig. 1C) and effective association constants (Fig. 1d) with a high correlation ( $86 \%$ and $99 \%$, respectively; Supplementary Figures $\mathbf{1 b}$ and $\mathbf{c}$ ). As validation, our model accurately captures a third, independent dataset of dCas9 effective association rates ${ }^{11}$ (Fig. 1e) with a correlation of $97 \%$ (Supplementary Figure 1d), and without the use of additional fitting parameters. Our model also predicts the CHAMP data for sequences with more than 2 mismatches, even though these were not included in the training data (Supplementary Figures $1 \mathbf{e}$ and $\mathbf{f}$ ). We conclude that our model accurately captures the physics of DNA binding and cleavage by Cas 9 .

a
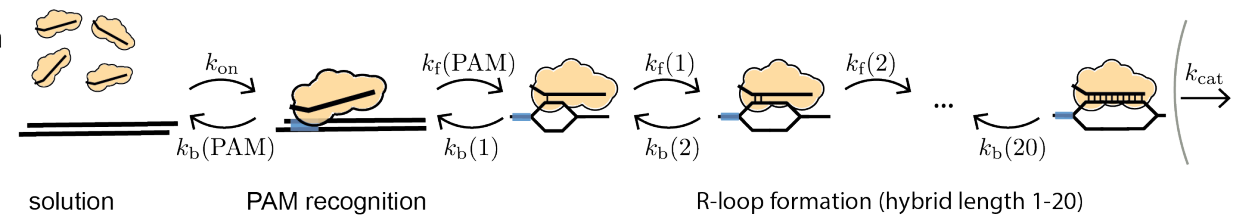

R-loop formation (hybrid length 1-20)

b

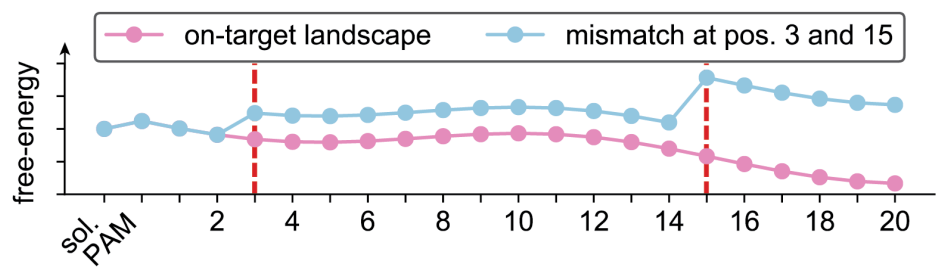

targeting progression

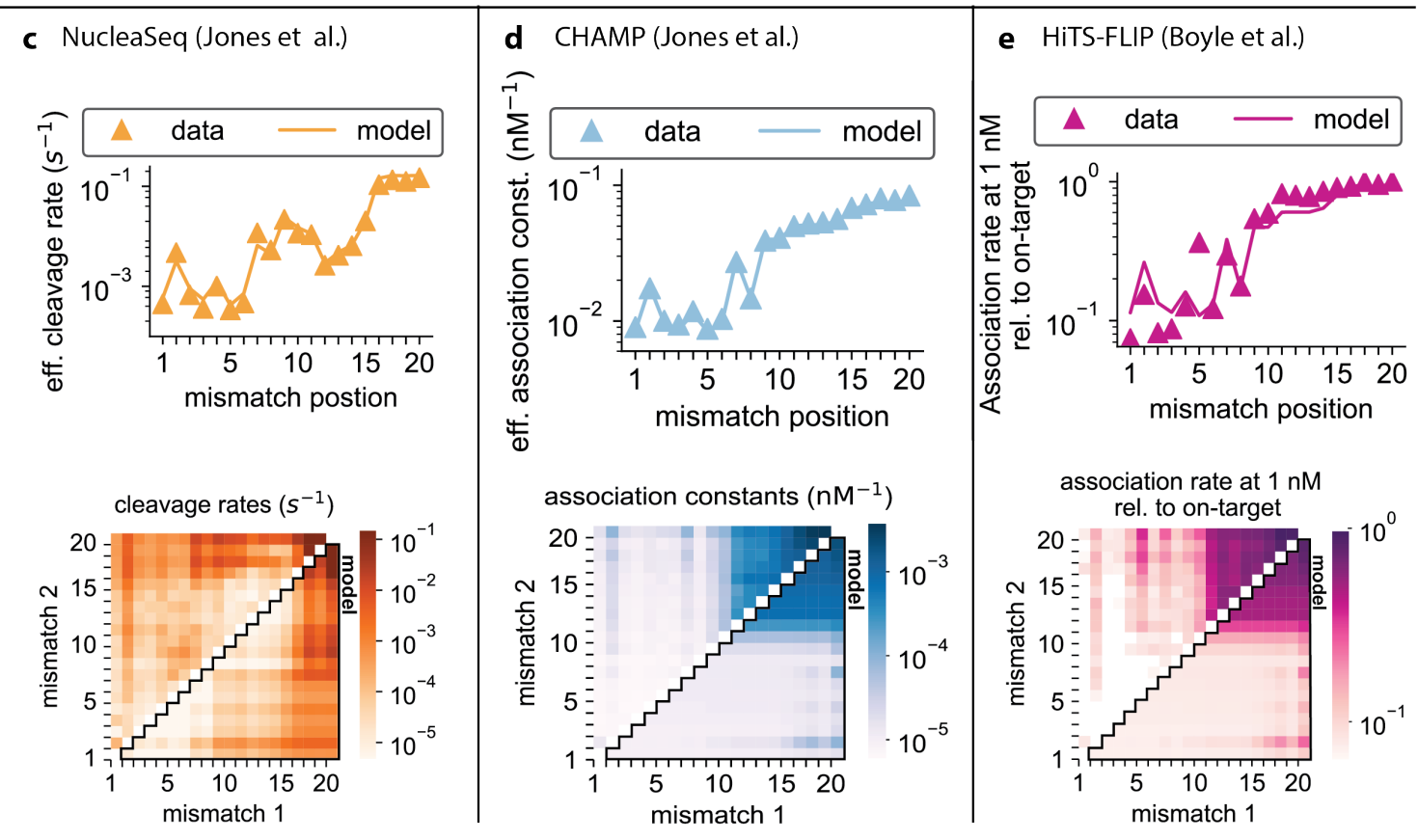

Fig. 1| A kinetic model captures both binding and cleavage data. a, Reaction schema underlying the proposed kinetic model (Supplementary Information for details). An available (d)Cas9-sgRNA from solution binds a DNA sequence (either on- or off-target) at its PAM site (blue rectangle) with rate $k_{\text {on }}$. R-loop formation then proceeds in one base-pair increments. A partially formed R-loop containing $n$ base pairs can either extend one base pair at a rate $k_{\mathrm{f}}(n)$ or shrink one base pair at a rate $k_{\mathrm{b}}(n)$. A complete R-loop (20 base pairs) is cleavage competent, and a dsDNA break is catalyzed at a rate $k_{\text {cat }}$. For dCas9, cleavage catalysis is not available, and $k_{\text {cat }}=0$. $\mathbf{b}$, Illustration of a possible free-energy landscape for Cas9-sgRNA-DNA for the on-target (pink) and an off-target with mismatches placed at positions 3 and 15 (blue). Each mismatch raises the entire free-energy landscape starting from the position where it occurs. c, Effective cleavage rates and $\mathbf{d}$, effective association constants as measured by and simultaneously fitted (Supplementary Information) to the NucleaSeq and CHAMP datasets (Jones at el.). Off-targets with one mismatch are show on top and off-targets with two mismatches are shown at the bottom (data above and model below diagonal), both as a function of mismatch position(s). e, Model prediction for effective binding rates as a function of mismatch position(s) compared to both HiTS-FLIP data and data from Boyle et al. top: one mismatch; bottom: two mismatches, with experimental data above and model results below the diagonal. 
Internal R-loop states are tuned for cleavage specificity without loss of on-target efficiency To gain mechanistic insights into the targeting reactions, we investigated the estimated free-energy landscape and kinetic parameters (Fig. 2) resulting from the simultaneous fit to our training datasets Figs. 1c,d). Starting from the PAM-bound state, the on-target free-energy (Fig. 2a) increases substantially when forming the first hybrid base pair and remains relatively high until the $8^{\text {th }}$ base pair is formed. This initial barrier must be bypassed before a stable binding intermediate is reached with about 11 or 12 hybridized base pairs. The freeenergy landscape reveals a second barrier to forming a full R-loop (13-18 bp), and eventual cleavage. The penalty for a mismatch (Fig. 2b) contains contributions from both DNA-RNA base pairing and proteinnucleotide interactions. Still, the mismatch penalties remain rather constant throughout $\left(6 \pm 1 k_{\mathrm{B}} T\right)$, with notable exceptions being positions 2, 18 and 20.

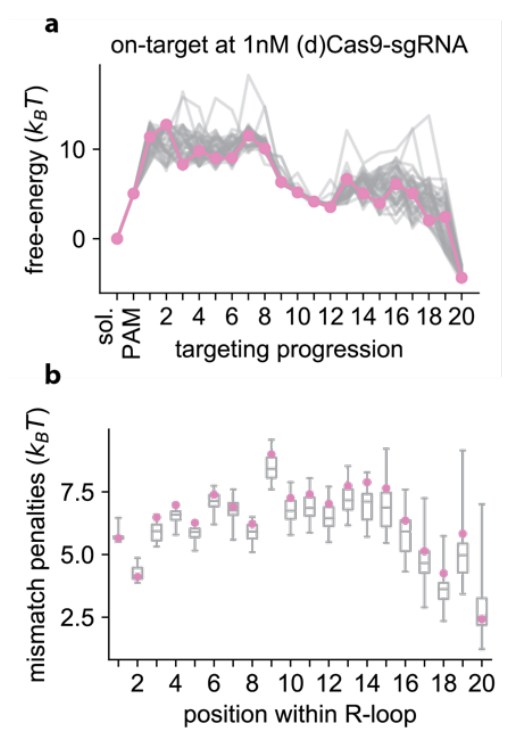

Fig. 2| Microscopic parameter estimated from NucleaSeq and CHAMP datasets. a, The free-energy landscape of the on-target reaction along the states shown in Fig. 1a. Here sol. is the solution state, PAM is the PAM-bound state and numbers indicate the number of R-loop base pairs formed. b, Energetic penalties for mismatches as a function of position. c, The three forward rates. In all panels, the global fit with the lowest chi-squared is shown in pink (Supplementary Information), and all nearly optimal solutions are represented in grey. For the lower two panels, the interquartile range of nearly optimal solutions are represented in the grey boxes and whiskers denote the complete range of values.

If the system equilibrates between major barriers in the free-energy landscape, we expect that any change in barrier height can be compensated for by the appropriate change in the bond-formation rate $\left(k_{\mathrm{f}}\right)$ (Supplementary Figures 2a,b)-without effecting model predictions. Consequently, both quantities cannot be simultaneously determined in a partially equilibrated system, explaining the high variability of predicted barrier heights (Fig. 2a) and $k_{\mathrm{f}}$ (Fig. 2c). By directly showing that the predicted binding and cleavage profiles are indeed insensitive to changing the barrier height (Supplementary Figures $\mathbf{2 c}$ and $\mathbf{d}$ ), as long as the forward rate is appropriately adjusted, we confirm partial equilibration of the system. This insight both explains the high variance of free-energy estimates in barrier regions (Fig. 2a), and allows us to perform coarse-grain modeling of the system to isolate parameters that are well determined by the data.

Based on the free-energy landscapes in Fig. 2a, we identified equilibrated states as those with free energies that are well-constrained by the fits. The equilibrated states are the effective states used in our coarse-grained model, and we calculate the coarse grained parametervalues based on the estimated parameter values of the full model (Supplementary Information). We define the open (O) R-loop state as the PAM bound state. The local minimum in Fig. 2a defines our coarse-grained intermediate (I) R-loop state with between 7 and 13 of its hybrid base pairs formed. Finally, the closed (C) R-loop and cleavage-competent state contains a fully formed hybrid. The resulting coarse-grained reaction scheme (Fig. 3a) captures the experimental data as well as the complete model (Supplementary Figure 3). This coarse-grained model reveals the rate-limiting steps during on- and offtarget DNA binding and cleavage.

The rate-limiting step for on-target cleavage is the transition from the open to the intermediate R-loop state $\left(k_{\mathrm{OI}} \ll k_{\mathrm{IC}}\right)$ (Figs. $\mathbf{3 b}$-d). Complexes that enter the intermediate state also typically enter the closed state $\left(k_{\mathrm{IO}} \ll k_{\mathrm{IC}}\right)$. The transition between the open and the intermediate state is reversible because the free-energy difference between the open and intermediate state is low (resulting in $k_{\mathrm{IO}} \approx k_{\mathrm{OI}}$ ). The free-energy difference between the intermediate and closed state is high $\left(k_{\mathrm{IC}} \gg k_{\mathrm{CI}}\right)$, rendering the transition from an opened to closed configuration essentially irreversible and all but guarantee cleavage $\left(k_{\mathrm{CI}} \ll k_{\text {cat }}\right)$. 
bioRxiv preprint doi: https://doi.org/10.1101/2020.05.21.108613; this version posted July 21, 2020. The copyright holder for this preprint (which was not certified by peer review) is the author/funder. All rights reserved. No reuse allowed without permission.

Mismatches between the target DNA and the sgRNA have differential effects on R-loop propagation. A PAMproximal seed mismatch (R-loop nucleotides 1-8) suppresses the rate of transition from an open to intermediate state $\left(k_{\mathrm{IO}}^{\text {on-target }} \gg k_{\mathrm{IO}}^{\text {seed m.m. }}\right.$ ) (Figs. 3e-g). In contrast, a PAM-distal mismatch (R-loop

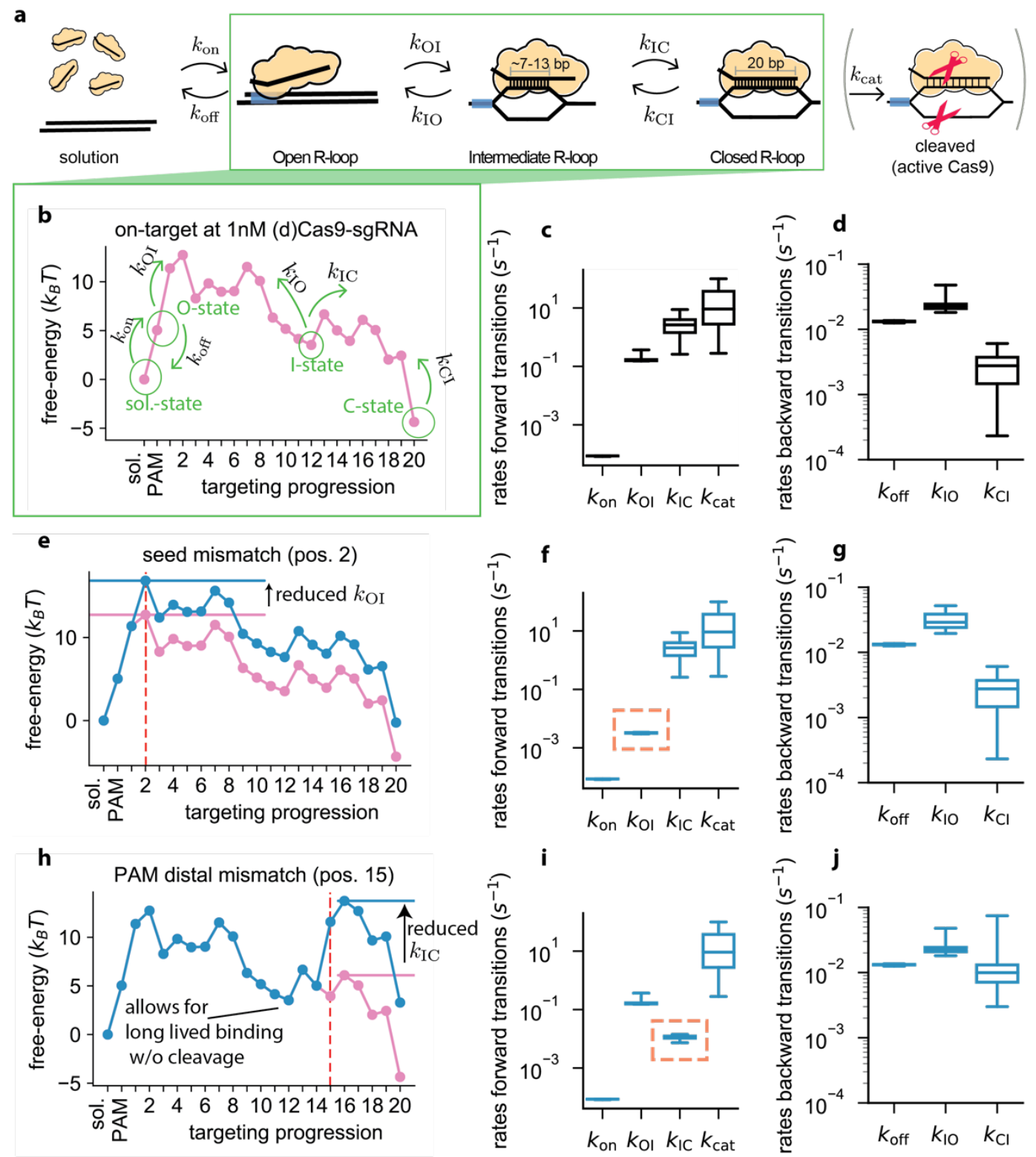

Fig. 3| Coarse-grained kinetic model fully captures bulk data. a, Coarse-grained version of the reaction scheme shown in Fig. 1a. Apart from the unbound and post-cleavage state, the targeting-reaction pathway is reduced to just three states (open, intermediate, and closed R-loops, see Supplementary Information for details). b, Microscopic free-energy landscape for the on-target exposed to 1nM (d)Cas9sgRNA (Fig. 2a) with coarse-grained states and rates indicated in green. c, Coarse-grained forward and d, backward rates associated with the landscape in b. e, Microscopic free-energy landscape for an off-target with a mismatch at position 2 exposed to 1nM (d)Cas9-sgRNA (blue), together with the on-target free-energy landscape (pink). $\mathbf{f}, \mathbf{g}$, Coarse-grained forward (f) and backward ( $\mathbf{g}$ ) rates associated with the landscape in $\mathbf{e}$. $\mathbf{h}$-j, Same as (f-g) for an off-target with a mismatch at position 15.

nucleotides 12-17) limits the effective rate of cleavage from the open state by reducing the intermediate to closed state transition $\left(k_{\mathrm{IC}} \ll k_{\mathrm{OI}}\right)$ (Figs. $\left.3 \mathrm{~h}-\mathrm{j}\right)$. The transition from binding to the intermediate state remains unaffected, though returning to the open state competes with completion of the R-loop $\left(k_{\mathrm{IO}} \approx k_{\mathrm{IC}}\right)$. Enzymes that enter the closed state likely also proceed to cleavage $\left(k_{\mathrm{CI}} \ll k_{\mathrm{cat}}\right)$. We conclude that specificity in PAMdistal regions (i.e., the second barrier in off-target landscape shown in Fig. $\mathbf{3 h}$ is higher than the first) is tuned not to interfere with the crucial on-target cleavage efficiency (i.e., second barrier in Fig. $\mathbf{3 b}$ is lower than the first). 
bioRxiv preprint doi: https://doi.org/10.1101/2020.05.21.108613; this version posted July 21, 2020. The copyright holder for this preprint (which was not certified by peer review) is the author/funder. All rights reserved. No reuse allowed without permission.

\section{R-loop propagation drives Cas9 conformation dynamics}

What are the structural properties of Cas9 that give rise to the non-monotonic free-energy landscape of Fig. 2a? A comparisons between DNA-bound and unbound Cas9-sgRNA structures have revealed that Cas9 repositions $\mathrm{HNH}$ and RuvC nuclease domains to catalyze cleavage ${ }^{44,53,54}$. We hypothesized that the position of the mobile $\mathrm{HNH}$ nuclease domain directly couples to R-loop progression, allowing it to influence its free-energy landscape. This hypothesis is based on the following key observations: First, ensemble FRET experiments ${ }^{49}$ detected two dominant Cas9 conformers with distinct HNH states, and single-molecule FRET studies have identified a third intermediate conformer ${ }^{40,41,51}$ - matching the number of R-loop states we find; Second, the relative position and occupancy of the $\mathrm{HNH}$ states is affected by R-loop mismatches ${ }^{40,41,51}$, while the Cas 9 can only sense mismatches by hybridizing the sgRNA with the target DNA.

a

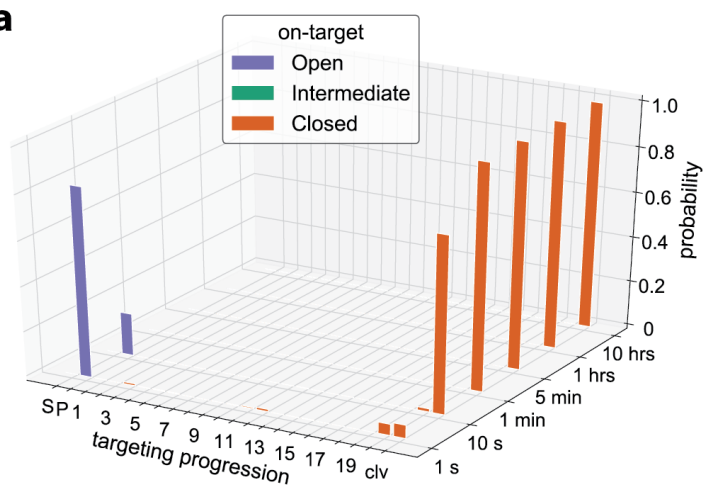

b

c

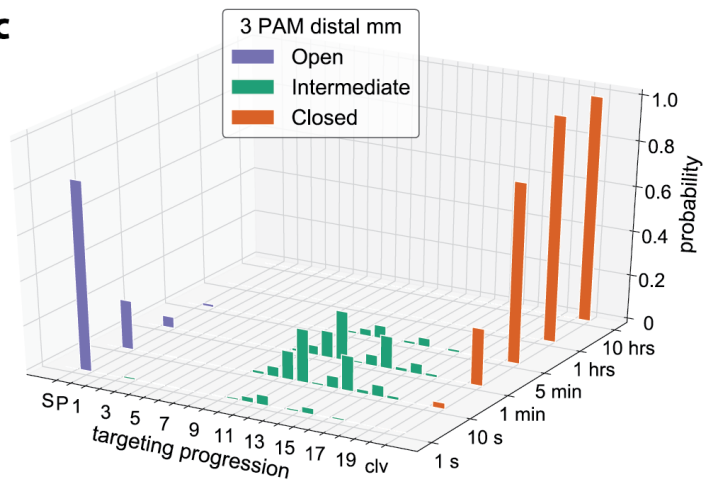

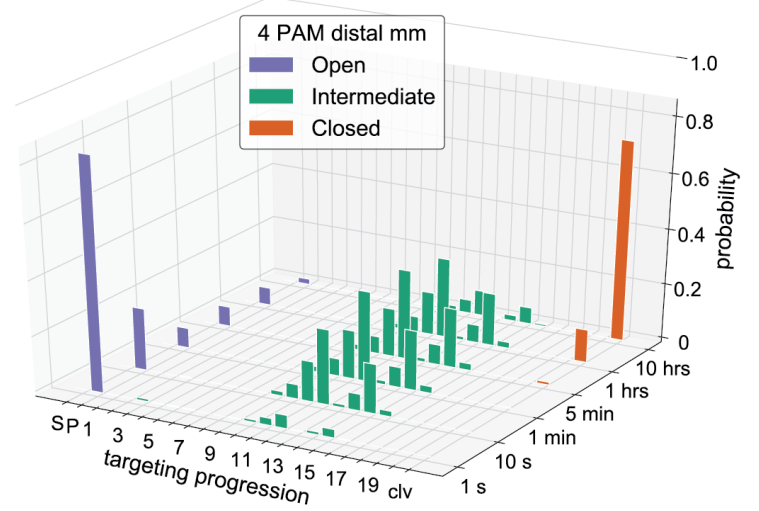

Fig. 4| The time evolution of R-loop hybridization is reminiscent of conformational dynamics. a, The evolution of the occupation probability for any of the 23 microscopic states shown in Fig. 1a, as a function of time when interacting with the on-target. $\mathbf{b}$, Same as a but when interacting with an off-target with last (PAM distal) 3 base pairs mismatched. c, Same as a but when interacting with an off-target with the last 4 base pairs mismatched. Colors indicate the corresponding coarse-grained R-loop configuration as defined in Fig. 3a: open R-loop and unbound states (blue), intermediate R-loop states (green) and cleavage-competent and post-cleavage states (orange).

To test this hypothesis, we mimicked the experiments of Dagdas et al. ${ }^{40}$ by calculating the time evolution of the occupancy for each of the microscopic states in the DNA-bound Cas9 landscape for three target sequences (Fig. 4). The HNH-domain completes its conformational change within seconds after Cas9-sgRNA binds to on-target $D_{N A}{ }^{40}$. Our model demonstrates a similar behavior for R-loop progression (Fig. 4a). The intermediate R-loop state (green) is visited only transiently, while the closed state (red) strongly resists unwinding of the full hybrid $\left(k_{\mathrm{IC}} \gg k_{\mathrm{CI}}\right)$ (Figs. 3c, d and 4a). Compared to the on-target DNA, PAM-distal mismatches reduce the intermediate closure rate $\left(k_{\mathrm{IC}}\right)$ and increase the time spent in the intermediate state (Fig. 4b), in agreement with prior observations ${ }^{40}$. Our model also shows how going from three to four PAM distal mismatches effectively abolishes the occupancy of the closed state at short times ${ }^{40}$, as R-loop formation is stalled in the intermediate state (Fig. 4c). In prior FRET experiments, the FRET value corresponding to the intermediate state depended on the number of mismatches introduced, which is evidence that the $\mathrm{HNH}$ domain adopts slightly different configurations $^{41}$. The reported relationship between FRET values and mismatches is consistent with tight coupling of conformational change to R-loop progression in PAM distal regions, as our model predicts that going from three to four PAM-distal mismatches increases the probability of residing as a larger intermediate R-loop (Fig. 4). Taken together, we propose that the three coarse-grained R-loop states identified in our freeenergy landscape reflect the three HNH domain conformers. The free-energy landscape (Fig. $\mathbf{2}$ and $\mathbf{3}$ ) obtained 
by fitting bulk data (Fig. 1) thus complements structural and single-molecule data to describe how Cas9 targets matched and mismatched DNAs.

\section{Kinetic modelling improves genome-wide off-target prediction}

Next, we sought to exploit our mechanistic description for predictive power, and compared our predictions with those of current state-of-the-art genomic off-target prediction tools. Current methods ${ }^{12,14,20,21,23-25,42}$ rank genomic off-targets according to various measures of in vivo activity without predicting biochemically measurable parameters (i.e., the binding affinity or cleavage rate). One such frequently-used tool computes the Cutting Frequency Determination (CFD) score ${ }^{12}$-a naïve-Bayes classification scheme ${ }^{22}$ that assumes mismatches affect the relative cleavage probabilities multiplicatively. More recently, Zhang et al. presented a unified CRISPR (UCRISPR) score that outperforms the CFD score ${ }^{24}$. uCRISPR estimates the cleavage probability as proportional to the Boltzmann weight corresponding to the cleavage competent state. The assumption of a multiplicative effect errors and the use of Boltzmann weights both implicitly imply binding equilibrium. This assumption is not borne out by the experimental data as the off-target binding and cleavage patterns do not match (see e.g. Supplementary Figure 1a).
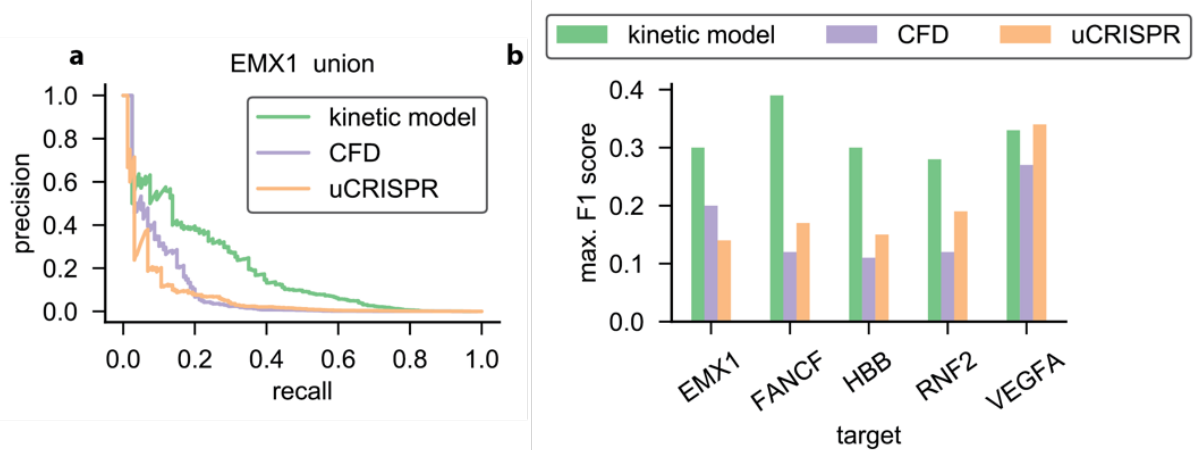

Fig. 5| Genome-wide off-target classification. a, Precision recall-curves for our model (green) and predictions based on the CFD score (purple) or uCRISPR score (orange) for the EMX1 site using all experimentally identified off-targets. b, F1-scores for our model (green), CFD prediction tool (purple) and uCRISPR (orange), for target sites EMX1, FANCF, HBB, RNF2 and VEGFA site 1 using all experimentally identified off-targets. For each condition, the maximum obtainable F1-score along the corresponding PR-curve is displayed (see a and Supplementary Figure 4)

To analyze whether our kinetic model improves genomic off-target predictions, we collected data from sequencing-based cleavage experiments. To comprehensively evaluate the model, we gathered data from all experiments that used industry-standard sgRNAs (i.e., targeting EMX1, FANCF, HBB, RNF2, and VEGFA) and reported multiple off-target cleavage sites ${ }^{33-36,38,39}$. Notably, these reports identified only partially overlapping sets of off-target cleavage sites, indicating that off-target cleavage detection is strongly dependent on experimental parameters (i.e., Cas9 nucleofection vs. plasmid transfection, exposure time, cell type, etc.) and the sensitivity of detection (i.e., enrichment of breaks or whole-genome sequencing) ${ }^{16,18}$. For each sgRNA, we separately tested against the union (sites found in any experiment) and intersection (sites found in every experiment) of the reported off-target sites (Fig. 5 and Supplementary Figures 4-6). The union of all reported off-targets maximizes the likelihood of covering low probability off-targets, while the intersection minimizes the effect of experiment-dependent biases and noise.

We tested how well our model, the CFD score, and UCRISPR separate reported off-targets over the human genome. For sake of comparison, we need to collapse our dynamic description into a binary classification. We choose to separate out strong off-targets based on the predicted cleavage vs. unbinding probability once the Cas9-sgRNA has bound the PAM $^{28}$, as this is proportional to the steady-state cleavage rate in the low concentration limit. To simplify the comparison further, we only considered sequences flanked by a canonical NGG motif. Fig. 5a shows the resulting precision-recall (PR) curve when tested against all reported off-targets of the EMX1 guide sequence (union). As the threshold for strong off-targets is swept, PR curves display the fraction of sites that are correctly labelled as off-target (precision) against the fraction of the experimentallyidentified sequences that are predicted (recall). For therapeutic genome-editing, a high recall is imperative as a false negative prediction is more harmful than a false positive one. Our kinetic model produces higher recall values for all achievable precisions, clearly outperforming state-of-the-art CFD and uCRISPR classifying schemes 
for EMX1 (Fig. 5a). Importantly, the kinetic model also outperforms the leading off-target predictors for highlymismatched genomic off-targets of other sgRNAs, as judged by PR-curves, receiver operating characteristic curves, and the F1-score (Fig. 5 and Supplementary Figures 4-6). This result is especially surprising since the kinetic model was trained on datasets that captured, at most, two mismatches from a single on-target sequence.

\section{Discussion}

Here, we describe a kinetic model for Cas9 binding and cleavage that is trained on high-throughput in vitro measurements ${ }^{15}$. This bottom-up modelling approach has allowed us to decipher the microscopic free-energy landscape underlying SpCas9 target recognition (Fig. 1-2). Based on extracted free-energy landscapes, we find that SpCas9's kinetics are dominated by transitions between the open, intermediate, and closed R-loop states (Fig. 3). As mismatches affect the three R-loop states similarly to the three configurational states of Cas9's nuclease domain $\mathrm{s}^{40,41}$, we propose that PAM distal R-loop formation is tightly coupled to protein conformation (Fig. 4)-pointing toward the relevant structure-function relation for the most important RNA-guided nuclease in use today.

By mechanistically accounting for the kinetic nature of the targeting process, our model outperforms existing genome-wide off-target prediction tools. For simplicity and robustness, we built our model to exclude mismatch type parameters, allowing for extensive training using datasets based on a single guide sequence and off-target DNAs containing up to two mismatches. This training does not limit the model's application as the model also improves on the detection of highly-mismatched genomic off-target sites (Fig. $\mathbf{5}$ and Supplementary Figures 4-6).

Our model is also the first to fully capture the time dependence of off-target binding in addition to cleavage. Understanding the time dependence of off-target binding will facilitate the design of sgRNA libraries in Cas9or dCas9-based experiments. For example, a recent study by Jost et al..$^{5}$ demonstrated that a series of mismatched guides can be used to titrate gene expression during CRISPRa/CRISPRi. Knowing SpCas9's microscopic free-energy landscape (Figs. 2-3) can also simplify the design of CRISPRa/CRISPRi libraries for novel gene targets. Wildtype Cas9 can also be (effectively) inactivated with PAM-distal mismatches in the guide ${ }^{55}$, and our model can guide titration of Cas9-sgRNA inactivation.

The physical insights generated by the free-energy landscapes we extract could also help rational proteinreengineering efforts aimed at producing high-fidelity Cas9 variants that maintain high on-target efficiency ${ }^{39,51,56}$. For SpCas9, we find that the barrier between the intermediate and closed states is tuned to extend the cleavage specificity beyond the seed, without affecting on-target efficiency (Figs. $\mathbf{3 b}$ and $\mathbf{h}$ ).

Taken together, we have shown that mechanistic modelling combined with high-throughput data sets give biophysical insights into SpCas9 off-targeting, and that those insights give predictive power far beyond the training sets. SpCas9 is only one of many RNA-guided nucleases with biotechnological applications, and other CRISPR associated nucleases (such as Cas12a, Cas13 and Cas14) offer a diversified genome-engineering toolkit ${ }^{15,57-62}$. These nucleases can all be characterized with our approach, and it will be especially interesting to compare the free-energy landscape of our SpCas9 benchmark to that of engineered ${ }^{39,51,56}$ and natural (e.g. N. meningitides Cas9 ${ }^{63}$ ) high-fidelity Cas9 variants.

\section{Acknowledgements}

We would like to thank Kristian Blom, Diewertje Dekker, and Sonny de Jong for valuable discussions and their help during the project. Thank you also to the members of the Chirlmin Joo lab and Stan Brouns lab for valuable discussions. We also thank Evan Boyle for sharing his data and answering all our questions. B.E.M. forms part of the research program "Crowd management: the physics of genome processing in complex environments", supported by NWO. M.K. was supported by the Netherlands Organization for Scientific Research (NWO/OCW), as part of the Frontiers in Nanoscience program. M.D. acknowledges support from the Parents in KIND program, sponsored by The Kavli Institute of Nanoscience Delft, the Department of Bionanoscience at TU Delft, and through a Spinoza Prize awarded to M. Dogterom by NWO. I.J.F. is supported by a University of Texas College of Natural Sciences Catalyst award and the Welch Foundation (F-1808). I.J.F. and S.K.J. are supported by the U.S. National Institute of Health (R01GM124141, F32AG053051). 


\section{Author contributions}

B.E.M and M.K: designed and performed the research. Wrote the manuscript K.v.d.S and C.v.d.S: Performed the research.

S.K.J: Provided data. Wrote manuscript

J.H: Provided data. Wrote manuscript

I.J.F: Provided data. Wrote manuscript

M.D: Designed the research. Wrote manuscript

\section{Competing Interests}

The authors declare no competing interests.

\section{References}

1. Sander, J. D. \& Joung, J. K. CRISPR-Cas systems for editing, regulating and targeting genomes. Nat. Biotechnol. 32, 347-350 (2014).

2. Wang, H., La Russa, M. \& Qi, L. S. CRISPR/Cas9 in Genome Editing and Beyond. Annu. Rev. Biochem. 85, 227-264 (2016).

3. Chen, B. et al. Dynamic imaging of genomic loci in living human cells by an optimized CRISPR/Cas system. Cell 155, 1479-1491 (2013).

4. Gilbert, L. A. et al. CRISPR-mediated modular RNA-guided regulation of transcription in eukaryotes. Cell 154, 442 (2013).

5. Jost, M. et al. Titrating gene expression using libraries of systematically attenuated CRISPR guide RNAs. Nat. Biotechnol. (2020). doi:10.1038/s41587-019-0387-5

6. Niu, D. et al. Inactivation of porcine endogenous retrovirus in pigs using CRISPR-Cas9. Science (80-. ). 1307, eaan4187 (2017).

7. Hammond, A. et al. A CRISPR-Cas9 gene drive system targeting female reproduction in the malaria mosquito vector Anopheles gambiae. Nat. Biotechnol. 34, 78-83 (2016).

8. Amoasii, L. et al. Gene editing restores dystrophin expression in a canine model of Duchenne muscular dystrophy. Science (80-. ). 362, 1-6 (2018).

9. Park, C. Y. et al. Functional Correction of Large Factor VIII Gene Chromosomal Inversions in Hemophilia A Patient-Derived iPSCs Using CRISPR-Cas9. Cell Stem Cell 17, 213-220 (2015).

10. Jinek, M. et al. A Programmable Dual-RNA - Guided. Science (80-. ). 337, 816-822 (2012).

11. Boyle, E. A. et al. High-throughput biochemical profiling reveals sequence determinants of dCas9 offtarget binding and unbinding. Proc. Natl. Acad. Sci. 114, 5461-5466 (2017).

12. Doench, J. G. et al. Optimized sgRNA design to maximize activity and minimize off-target effects of CRISPR-Cas9. Nat. Biotechnol. 34, 184-191 (2016).

13. Fu, Y. et al. High-frequency off-target mutagenesis induced by CRISPR-Cas nucleases in human cells. Nat. Biotechnol. 31, 822-826 (2013).

14. Hsu, P. D. et al. DNA targeting specificity of RNA-guided Cas9 nucleases. Nat. Biotechnol. 31, 827-832 (2013).

15. Jones Jr, S. K. et al. Massively parallel kinetic profiling of natural and engineered CRISPR nucleases. BioRxiv 1-17 (2019).

16. Kim, D., Luk, K., Wolfe, S. A. \& Kim, J.-S. Evaluating and Enhancing Target Specificity of Gene-Editing 
Nucleases and Deaminases. Annu. Rev. Biochem. 1-30 (2019). doi:10.1146/annurev-biochem-013118111730

17. Pattanayak, V. et al. High-throughput profiling of off-target DNA cleavage reveals RNA-programmed Cas9 nuclease specificity. Nat. Biotechnol. 31, 839-843 (2013).

18. Tsai, S. Q. \& Joung, J. K. Defining and improving the genome-wide specificities of CRISPR-Cas9 nucleases. Nat. Rev. Genet. 17, 300-312 (2016).

19. Cullot, G. et al. CRISPR-Cas9 genome editing induces megabase-scale chromosomal truncations. Nat. Commun. 10, 1-14 (2019).

20. Labun, K., Montague, T. G., Gagnon, J. A., Thyme, S. B. \& Valen, E. CHOPCHOP v2: a web tool for the next generation of CRISPR genome engineering. Nucleic Acids Res. 44, W272-W276 (2016).

21. Heigwer, F., Kerr, G. \& Boutros, M. E-CRISP: Fast CRISPR target site identification. Nat. Methods 11, 122-123 (2014).

22. Listgarten, J. et al. Prediction of off-target activities for the end-to-end design of CRISPR guide RNAs. Nat. Biomed. Eng. 2, 38-47 (2018).

23. Chuai, G. et al. DeepCRISPR: Optimized CRISPR guide RNA design by deep learning. Genome Biol. 19, 118 (2018).

24. Zhang, D., Hurst, T., Duan, D. \& Chen, S.-J. Unified energetics analysis unravels SpCas9 cleavage activity for optimal gRNA design. Proc. Natl. Acad. Sci. 116, 8693-8698 (2019).

25. Stemmer, M., Thumberger, T., Del Sol Keyer, M., Wittbrodt, J. \& Mateo, J. L. CCTop: An intuitive, flexible and reliable CRISPR/Cas9 target prediction tool. PLoS One 10, 1-11 (2015).

26. Tycko, J., Myer, V. E. \& Hsu, P. D. Methods for Optimizing CRISPR-Cas9 Genome Editing Specificity. Mol. Cell 63, 355-370 (2016).

27. Farasat, I. \& Salis, H. M. A Biophysical Model of CRISPR/Cas9 Activity for Rational Design of Genome Editing and Gene Regulation. PLoS Comput. Biol. 12, 1-33 (2016).

28. Klein, M., Eslami-Mossallam, B., Arroyo, D. G. \& Depken, M. Hybridization Kinetics Explains CRISPR-Cas Off-Targeting Rules. Cell Rep. 22, (2018).

29. Bisaria, N., Jarmoskaite, I. \& Herschlag, D. Lessons from Enzyme Kinetics Reveal Specificity Principles for RNA-Guided Nucleases in RNA Interference and CRISPR-Based Genome Editing. Cell Syst. 4, 21-29 (2017).

30. O'Geen, H., Henry, I. M., Bhakta, M. S., Meckler, J. F. \& Segal, D. J. A genome-wide analysis of Cas9 binding specificity using ChIP-seq and targeted sequence capture. Nucleic Acids Res. 43, 3389-3404 (2015).

31. Wu, X. et al. Genome-wide binding of the CRISPR endonuclease Cas9 in mammalian cells. Nat. Biotechnol. 32, 670-676 (2014).

32. Kuscu, C., Arslan, S., Singh, R., Thorpe, J. \& Adli, M. Genome-wide analysis reveals characteristics of offtarget sites bound by the Cas9 endonuclease. Nat. Biotechnol. 32, 677-683 (2014).

33. Cameron, P. et al. Mapping the genomic landscape of CRISPR-Cas9 cleavage. Nat. Methods 14, 600606 (2017).

34. Tsai, S. Q. et al. CIRCLE-seq: A highly sensitive in vitro screen for genome-wide CRISPR-Cas9 nuclease off-targets. Nat. Methods 14, 607-614 (2017).

35. Kim, D. et al. Digenome-seq: Genome-wide profiling of CRISPR-Cas9 off-target effects in human cells. 
Nat. Methods 12, 237-243 (2015).

36. Tsai, S. Q. et al. GUIDE-seq enables genome-wide profiling of off-target cleavage by CRISPR-Cas nucleases. Nat. Biotechnol. 33, 187-198 (2015).

37. Frock, R. L. et al. Genome-wide detection of DNA double-stranded breaks induced by engineered nucleases. Nat. Biotechnol. 33, 179-188 (2015).

38. Yan, W. X. et al. BLISS is a versatile and quantitative method for genome-wide profiling of DNA doublestrand breaks. Nat. Commun. 8, 1-9 (2017).

39. Slaymaker, I. M. et al. Rationally engineered Cas9 nucleases with improved specificity. Science (80-. ). 351, 84-88 (2016).

40. Dagdas, Y. S., Chen, J. S., Sternberg, S. H., Doudna, J. A. \& Yildiz, A. A conformational checkpoint between DNA binding and cleavage by CRISPR-Cas9. Sci. Adv. 3, 1-9 (2017).

41. Yang, M. et al. The Conformational Dynamics of Cas9 Governing DNA Cleavage Are Revealed by SingleMolecule FRET. Cell Rep. 22, 372-382 (2018).

42. Haeussler, M. et al. Evaluation of off-target and on-target scoring algorithms and integration into the guide RNA selection tool CRISPOR. Genome Biol. 17, 1-12 (2016).

43. Anders, C., Niewoehner, O., Duerst, A. \& Jinek, M. Structural basis of PAM-dependent target DNA recognition by the Cas9 endonuclease. Nature 513, 569-573 (2014).

44. Jiang, F., Zhou, K., Gressel, S. \& Doudna, J. A. A cas9 guide RNA complex preorganized for target DNA recognition. Science (80-. ). 348, 1477-1482 (2015).

45. Josephs, E. A. et al. Structure and specificity of the RNA-guided endonuclease Cas9 during DNA interrogation, target binding and cleavage. Nucleic Acids Res. 43, 8924-8941 (2015).

46. Rutkauskas, M. et al. Directional R-loop formation by the CRISPR-cas surveillance complex cascade provides efficient off-target site rejection. Cell Rep. 10, 1534-1543 (2015).

47. Szczelkun, M. D. et al. Direct observation of R-loop formation by single RNA-guided Cas9 and Cascade effector complexes. Proc. Natl. Acad. Sci. 111, 9798-9803 (2014).

48. Xiao, Y. et al. Structure Basis for Directional R-loop Formation and Substrate Handover Mechanisms in Type I CRISPR-Cas System. Cell 170, 48-60.e11 (2017).

49. Sternberg, S. H., Lafrance, B., Kaplan, M. \& Doudna, J. A. Conformational control of DNA target cleavage by CRISPR-Cas9. Nature 527, 110-113 (2015).

50. Sung, K., Park, J., Kim, Y., Lee, N. K. \& Kim, S. K. Target Specificity of Cas9 Nuclease via DNA Rearrangement Regulated by the REC2 Domain. J. Am. Chem. Soc. 140, 7778-7781 (2018).

51. Chen, J. S. et al. Enhanced proofreading governs CRISPR-Cas9 targeting accuracy. Nature 550, 407-410 (2017).

52. Jung, C. et al. Massively Parallel Biophysical Analysis of CRISPR-Cas Complexes on Next Generation Sequencing Chips. Cell 170, 35-47.e13 (2017).

53. Jiang, F. et al. Structures of a CRISPR-Cas9 R-loop complex primed for DNA cleavage. Science (80-. ). 351, 867-871 (2016).

54. Jinek, M. et al. Structures of Cas9 endonucleases reveal RNA-mediated conformational activation. Science (80-. ). 343, (2014).

55. Dahlman, J. E. et al. Orthogonal gene knockout and activation with a catalytically active Cas9 nuclease. 
Nat. Biotechnol. 33, 1159-1161 (2015).

56. Kleinstiver, B. P. et al. High-fidelity CRISPR-Cas9 nucleases with no detectable genome-wide off-target effects. Nature 529, 490-495 (2016).

57. Chen, J. S. et al. CRISPR-Cas12a target binding unleashes indiscriminate single-stranded DNase activity. Science (80-. ). 360, 436-439 (2018).

58. Gootenberg, J. S. et al. Nucleic acid detection with CRISPR-Cas13a/C2c2. Science 356, 438-442 (2017).

59. Gootenberg, J. S. et al. Multiplexed and portable nucleic acid detection platform with Cas13, Cas12a, and Csm6. Science (80-. ). 444, 439-444 (2018).

60. Harrington, L. B. et al. Programmed DNA destruction by miniature CRISPR-Cas14 enzymes. Science (80.). 362, 839-842 (2018).

61. Kim, D. et al. Genome-wide analysis reveals specificities of Cpf1 endonucleases in human cells. Nat. Biotechnol. 34, 863-868 (2016).

62. Kleinstiver, B. P. et al. Genome-wide specificities of CRISPR-Cas Cpf1 nucleases in human cells. Nat. Biotechnol. 34, 869-874 (2016).

63. Amrani, N. et al. NmeCas9 is an intrinsically high-fidelity genome-editing platform Jin-Soo Kim. Genome Biol. 19, 1-25 (2018). 
bioRxiv preprint doi: https://doi.org/10.1101/2020.05.21.108613; this version posted July 21, 2020. The copyright holder for this preprint (which was not certified by peer review) is the author/funder. All rights reserved. No reuse allowed without permission. 